\title{
The apparency hypothesis applied to a local pharmacopoeia in the Brazilian northeast
}

\author{
Alejandro Lozano ${ }^{1}$, Elcida Lima Araújo², Maria Franco Trindade Medeiros ${ }^{3}$ and Ulysses Paulino Albuquerque ${ }^{*}$
}

\begin{abstract}
Background: Data from an ethnobotanical study were analyzed to see if they were in agreement with the biochemical basis of the apparency hypothesis based on an analysis of a pharmacopeia in a rural community adjacent to the Araripe National Forest (Floresta Nacional do Araripe - FLONA) in northeastern Brazil. The apparency hypothesis considers two groups of plants, apparent and non-apparent, that are characterized by conspicuity for herbivores (humans) and their chemical defenses.

Methods: This study involved 153 interviewees and used semi-structured interviews. The plants were grouped by habit and lignification to evaluate the behavior of these categories in terms of ethnospecies richness, use value and practical and commercial importance. Information about sites for collecting medicinal plants was also obtained. The salience of the ethnospecies was calculated. G-tests were used to test for differences in ethnospecies richness among collection sites and the Kruskal-Wallis test to identify differences in the use values of plants depending on habit and lignifications (e.g. plants were classes as woody or non-woody, the first group comprising trees, shrubs, and lignified climbers (vines) and the latter group comprising herbs and non-lignified climbers). Spearman's correlation test was performed to relate salience to use value and these two factors with the commercial value of the plants.
\end{abstract}

Results: A total of 222 medicinal plants were cited. Herbaceous and woody plants exhibited the highest ethnospecies richness, the non-woody and herbaceous plants had the most practical value (current use), and anthropogenic areas were the main sources of woody and non-woody medicinal plants; herbs and trees were equally versatile in treating diseases and did not differ with regard to use value. Trees were highlighted as the most commercially important growth habit.

Conclusions: From the perspective of its biochemical fundamentals, the apparency hypothesis does not have predictive potential to explain the use value and commercial value of medicinal plants. In other hand, the herbaceous habit showed the highest ethnospecies richness in the community pharmacopeia, which is an expected prediction, corroborating the apparency hypothesis.

\section{Background}

When applied from the ethnobotanical perspective, the apparency hypothesis, which was developed by ecologists in the 1970s (see [1,2]), treats plants as resources and humans as herbivores or consumers that require these resources [3-6]. This hypothesis considers two groups of plants, apparent and non-apparent, that are

\footnotetext{
* Correspondence: upa@db.ufrpe.br

'Departamento de Biologia, Área de Botânica, Laboratório de Etnobiologia Aplicada e Teórica (LEA), Universidade Federal Rural de Pernambuco, Rua Dom Manoel de Medeiros s/n, Dois Irmãos, Recife 52171-030, Pernambuco, Brasil

Full list of author information is available at the end of the article
}

characterized by their conspicuity (apparency) for herbivores. Non-apparent plants are difficult for herbivores to find because the plants are not predictably distributed in space and time; this group includes herbs, short-cycle plants, and those at early stages of plant succession $[3,5]$. Apparent plants are perennial, woody plants that usually dominate forest ecosystems and are comparatively more predictable in space and time, thus being easily detected by herbivores $[3,5]$. In general, one could characterize non-woody plants (herbs and non-woody climbers) as non-apparent and woody ones (trees, shrubs and vines) as apparent. From the biochemical perspective of antiherbivore defense, non-apparent plants are characterized

\section{Biomed Central}


by producing qualitative defenses, such as highly bioactive, low molecular weight compounds at low concentrations (e.g., alkaloids and terpenoids), whereas apparent plants are characterized by producing digestion-reducing quantitative defenses (tannins and lignins) [3]. Apparent and non-apparent are relative terms and must be applied according to the scenario to be investigated. For example, according to one reviewer of this paper "pioneer vegetation consisting of herbs (here called non-apparent plants) is apparent immediately after human disturbance (during early successional stages)".

The apparency hypothesis relates to plant succession in that plants characterized as non-apparent dominate the early stages of plant succession, whereas plants characterized as apparent dominate forests in later successional stages [3]. Studies conducted by different authors have shown that secondary vegetation is the main source of medicinal plants for tropical pharmacopoeias [7-9], highlighting the importance of herbs and exotic plants in some cases [10-12]. Exotic plants are related to plant succession because they usually establish themselves in disturbed habitats (secondary vegetation or cultivated areas) and most often exhibit an herbaceous habit [11]. Voeks [13] observed the importance of secondary vegetation, herbaceous habit and/or exotic species in the pharmacopoeias of different sites and concluded that tropical pharmacopoeias are primarily a product of disturbed sites that are covered by secondary vegetation.

Indices that quantify the relative value of plant species for people are required for testing the apparency hypothesis. The use value, initially presented by Phillips and Gentry [14], and relative importance, proposed by Bennett and Prance [10], are among the indices usually used for this purpose. Both of these indices are characterized by quantifying people's knowledge of plants, but neither indicates whether the plants are actually being used [15], which limits the interpretation of their results beyond the cognitive domain. This difference between knowledge of the potential and actual use of particular plants $[15,16]$ indicates that species with high use values do not necessarily have high practical values (current use).

Few studies have sought to ascertain whether medicinal plants belonging to secondary vegetation are actually used by the people who cite them [15]. According to the apparency hypothesis, secondary vegetation zones would be important sources of medicinal plants containing highly bioactive compounds [6,9,11,17]; therefore, one would expect these plants to be culturally important (high use value), widely used by local populations (high practical value) [15] and highlighted in the local market.

In this paper, the local pharmacopeia from a community adjacent to the Araripe National Forest (Floresta Nacional de Araripe - FLONA-Araripe) in northeastern Brazil is analyzed using habit and lignification as two means of grouping plants. The aim is to discuss the apparency hypothesis and answer the following questions: (1) Does medicinal plant richness vary between habits and between woody and non-woody plants? (2) Does contribution in terms of medicinal plant richness vary among collection sites? (3) Do use value and commercial and practical importance varies between medicinal plant habits and between woody and non-woody plants? Using the biochemical fundamentals of the apparency hypothesis, it would be expected that (1) there is a higher richness of non-woody and herbaceous medicinal species as well as (2) a higher contribution of medicinal plants from anthropogenic zones. It would also be expected that (3) non-woody and herbaceous species stand out in cognitive, practical, and commercial importance metrics.

\section{Methods}

\section{Study area}

The Araripe National Forest or FLONA-Araripe was established in 1946 and currently encompasses an area of 38,262.33 hectares. Most of this area is above the 'Chapada do Araripe' [Araripe Plateau], a formation that reaches $900 \mathrm{~m}$ in altitude, where the annual rainfall ranges between 671 and 2,291 $\mathrm{mm}$ and the average temperature is $23^{\circ} \mathrm{C}$ [18]. Most of the vegetation within this conservation unit is 'Cerrado' [wooded savannah] (48.53\%), followed by 'Cerradão' [densely wooded savannah] (27.49\%), rainforest (22.47\%), and 'Carrasco' [xerophytic shrubby vegetation] (1.51\%) [18].

The study was conducted in the rural community of Horizonte $\left(07^{\circ} 29^{\prime} 36.9^{\prime \prime} \mathrm{S}, 39^{\circ} 22^{\prime} 06.02^{\prime \prime} \mathrm{W}\right)$ adjacent to FLONA-Araripe, located in the municipality of Jardim, Cariri region, Ceará State in northeastern Brazil. Horizonte has a registered population of 1,120 inhabitants (based on census information obtained by the community's health workers).

Currently, there is a health-care center in Horizonte to which the population has access through consultations every Monday, when a doctor evaluates patients and provides some pharmaceutical drugs.

The residents primarily conduct subsistence agriculture. The crops typically grown include beans, cassava, and maize; only occasionally, namely when harvests are abundant, is the surplus sold. Cattle-raising activity does not generate high revenues within the region because it is conducted in the form of a small-scale production system with very low zootechnical indices [18].

The extraction of non-timber forest products is a major source of income for the area, with approximately $60 \%$ of the population over 18 claiming to do so. Resources are collected from within the FLONA and also, to a lesser extent, from private properties of the Horizonte residents. Among the species collected, 'pequi' 
(Caryocar coriaceum Wittm.) and the 'fava d'anta' tree (Dimorphandra gardneriana Tul.) stand out in terms of profit.

\section{Consent}

The objectives of the project, along with the methodological procedures employed, were previously presented and discussed with the members of the community studied here, who were then asked to agree to participate and to sign a Free and Transparent Consent form (TCLE).

\section{Data collection}

The Biodiversity Authorization and Information System (Sistema de Autorização e Informação em Biodiversidade - SISBIO) granted authorization for the present scientific activities (authorization number 27440-1), and the research ethics committee (Conselho de Ética em Pesquisa - Plataforma Brasil) provided authorization number 251.749 with CAAE 02845312.0.0000.5207 for developing this study.

Semi-structured interviews were conducted in a intensive fieldwork between March and May 2011 to collect information on medicinal plants. A total of 153 people over 18 years of age were interviewed after being randomly selected (using random sampling without replacement) from a total of 462 people in the community (according to the Department of Health census). Of the 153 interviews, 93 were with women (18 to 86 years old) and 60 were with men (18 to 80 years old). The time and place of each interview was scheduled according to each interviewee's availability [19]. Data were checked and supplemented in the years 2012 and 2013.

The first phase of each interview was to develop a free list of the medicinal plants known to the informants and use complementary techniques, such as rereading and asking related questions (non-specifically induced) on the topic, to enrich the lists $[17,19]$. Once the list was completed, the interviewee was asked to mention other names that he or she knew for each of the plants previously mentioned as well as collection sites, parts used, therapeutic indications, whether the plants had any commercial value, and which parts were intended to be sold. Furthermore, the respondent was asked to identify the medicinal plants that he or she had used within the past month to obtain information on the actual use and thus the practical value of the plants; finally, the interviewee was asked to identify a resident who was knowledgeable of medicinal plants within their community.

The number of plants mentioned in the free lists was used as a quantitative criterion for choosing knowledgeable locals [19]. By combining the information in the free lists with the names of locals identified in the interviews as being knowledgeable of medicinal plants, it was possible to obtain a list of people (within the group of those randomly selected) who were best suited to participate in the study as local experts on medicinal plants. Five people were chosen as local experts: two men (63 and 48 years old) and three women $(61,44$, and 31 years old), and they led individual guided tours to collect the medicinal plants mentioned in the interviews that occur within the region. A botanical sample was associated with a common name selected based on consensus among the locals experts.

During the botanical collections, the habits of the plants were identified according to the parameters proposed by Begon et al. [20] and adapted to the local vegetation according to Costa and Araújo [21]. Thus, woody perennials that usually had only one stem or that consistently branched at least $50 \mathrm{~cm}$ above the ground were classified as trees, woody perennials that were smaller and exhibited abundant branching or branches consistently below $50 \mathrm{~cm}$ were classified as shrubs, nonlignified plants or those only lignified at the base of the stem were classified as herbs, and woody or non-woody plants that used the structure of other plants for support were classified as climbers. Next, the plants were classes as woody or non-woody, the first group comprising trees, shrubs, and lignified climbers (vines) and the latter group comprising herbs and non-lignified climbers. In addition, the plants were also grouped according to their origin: plants that originated from outside of South America were considered to be exotic plants, and those from South America were considered natives.

The botanical collections were conducted during May and June 2011 and supplemented with collections from our team (Laboratory of Applied and Theoretical Ethnobiology) between the years 2012 and 2013. All the specimens were identified (according to APG [Angiosperm Phylogeny Group] III) and deposited at the Dárdano de Andrade and Lima Herbarium (Herbário Caririense Dárdano de Andrade e Lima - HCDAL) of the Regional University of Cariri (Universidade Regional do Cariri - URCA) in the city of Crato, Ceará. The voucher numbers run from HCDAL 6558 to 6701 and from 8104 to 8110 . Duplicates were deposited at the Professor Vasconcelos Sobrinho herbarium (PEUFR) of the Federal Rural University of Pernambuco (Universidade Federal Rural de Pernambuco- UFRPE), Recife, Pernambuco.

\section{Data analysis}

The plants were grouped by habit and lignification to test the predictions derived from the apparency hypothesis for the various aspects of the pharmacopeia analyzed.

This study used the local classification of the plants, defining as an ethnospecies each taxonomic entity considered by the interviewees. Regarding the collection 
sites, an ethnospecies was considered to originate from another region if $75 \%$ or more of the 153 interviewees cited it in any of the following categories: commercially traded, brought from the 'Sertão' (Caatinga vegetation, tropical dry forest of Northeastern Brazil surrounding the 'Chapada do Araripe') or brought from the 'Cariri' (low areas down the 'Chapada').

The collection sites in the FLONA region were grouped into two major types: anthropogenic areas (backyards, farms, swiddens, roadsides, paddocks, etc.) and native vegetation (vegetation present around the community on private land and vegetation from the FLONA). Each ethnospecies received a certain percentage of citations for these two types of collection sites. This percentage was used to analyze the ethnospecies richness per collection site type, determined as follows: ethnospecies with two or more informants and $75 \%$ or more citations for the site, ethnospecies with two or more informants and less than $75 \%$ citations for the site, and those with a single informant. The native vegetation was later separated into the areas outside and within the FLONA to further understand the conservation unit's role as a collection site for medicinal plants.

The plants mentioned as being used during the month preceding the interview (recently used) were analyzed to obtain information about the plants' practical importance in relation to habit and lignification.

The use value (treated here as theoretical knowledge) was calculated according to the modified version proposed by Rossato et al. [22], in which $\mathrm{VU}=\Sigma \mathrm{U} i / \mathrm{n}$, where $\mathrm{U} i=$ the number of uses mentioned by each informant for a given species and $n=$ the total number of informants. Only the principal use for which is employed each ethnospecies is reported within this manuscript, that is, the use that received more citations from the interviewers.

The commercial value was expressed as the percentage of citations affirming sale inside and/or outside of the village. The plants considered to be commercially important were those for which more than $75 \%$ of the total interviewees stated that the plant is sold (plants with only one citation were excluded).

Using Anthropac 4.0 software [23], the salience of the ethnospecies mentioned in the free lists of medicinal plants was calculated. Two software programs were used for statistical analyses. BioEstat 5.0 for Windows [24] was used to conduct the G-test to test for differences in ethnospecies proportion among collection sites and the Kruskal-Wallis test to identify differences in the use values of the plants depending on habit and lignifications. Using SPSS 17.0, Spearman's correlation test was performed to relate salience to use value and these two factors to the commercial value of the plants.

\section{Results and discussion}

\section{Ethnospecies richness per habit}

A total of 222 ethnospecies were mentioned in the free lists. Of the 222 ethnospecies, 44 were considered to come from other regions, and of the 178 ethnospecies collected from the FLONA region, 62 were herbs (36.9\%), 46 were trees $(27.4 \%), 46$ were shrubs $(27.4 \%), 13$ were climbers $(7.7 \%)$, and there was one parasite $(0.6 \%)$ (Table 1$)$. Ten plants were neither collected nor classified according to their habit due to difficulty in finding them in the forest or to no longer being found in backyards of the community; these plants had low numbers of citations, between one and five at the most. The herbaceous habit showed the highest ethnospecies richness in the community pharmacopeia, which is an expected result, corroborating the apparency hypothesis from the perspective of biochemical fundamentals.

In grouping the plants as woody and non-woody, we obtained 71 non-woody (42.3\%) and 97 woody plants (57.7\%), the latter group having the most ethnospecies, which is not in accordance with the expected results. Thus, it is clear that the type of classification used to characterize the plants defines whether the findings corroborate the predictions of the hypothesis. Because lignification is an attribute that defines apparent and non-apparent plants $[3,4]$, the classification of plants as woody and non-woody is more suited for the apparency hypothesis; furthermore, it is a dichotomous grouping with two mutually exclusive groups. Despite this, the two classification types clearly define the groups that represent non-apparent plants (herbs and non-woody) and allow testing of the predictions made in the present study.

The dominance of herbaceous plants in terms of medicinal species richness has also been reported in pharmacopoeias from other Brazilian ecosystems, such as the Caatinga [semi-arid vegetation] [25] and Atlantic Forest [12], and also in other locations such as Southern Mexico [11]. In the study by Albuquerque et al. [25], woody (trees, shrubs, and sub-shrubs) and non-woody (herbaceous) plants totaled $56.6 \%$ and $43.4 \%$ of medicinal plants, respectively, proportions similar to those found in the present study, showing that woody plants exhibited the higher medicinal species richness. In contrast, in the study by Voeks [12], using either of the two types of grouping proposed in the present study, the prediction of dominance by non-woody (herbaceous) plants based on the biochemical fundamentals of the apparency hypothesis was corroborated. In that study, woody (trees and shrubs) and non-woody plants (herbs and epiphytes) totaled $35 \%$ and $56 \%$ of the medicinal plants, respectively (lianas, the remaining 9\%, were not included because they are both woody and non-woody). This result indicates that non-woody medicinal plants, specifically 
Table 1 Medicinal plants mentioned in the free lists and collected by the locals within the FLONA region (Horizonte community, NE Brazil)

\begin{tabular}{|c|c|c|c|c|c|c|c|c|}
\hline Family/Scientific name & Ethnospecies & Principal use & Habit & Origin & $\begin{array}{l}\text { Use } \\
\text { value }\end{array}$ & Salience & $\begin{array}{l}\text { Commercial } \\
\text { value }\end{array}$ & Voucher \\
\hline \multicolumn{9}{|l|}{ AMARANTHACEAE } \\
\hline $\begin{array}{l}\text { Alternanthera brasiliana (L.) } \\
\text { Kuntze }\end{array}$ & nelvagina & headache & herb & native & 0.235 & 0.102 & 0.0 & n.c \\
\hline Chenopodium ambrosioides L. & mentruz & bone fracture & herb & exotic & 0.497 & 0.137 & 7.7 & n.c \\
\hline \multicolumn{9}{|l|}{ ANACARDIACEAE } \\
\hline Anacardium occidentale $\mathrm{L}$. & cajueiro & healing wounds & tree & native & 0.418 & 0.146 & 47.7 & n.c \\
\hline Mangifera indica $\mathrm{L}$. & mangueira & breathing difficulties & tree & exotic & 0.020 & 0.009 & 0.0 & n.c \\
\hline $\begin{array}{l}\text { Myracrodruon urundeuva } \\
\text { Allemão }\end{array}$ & aroeira & cutaneous illness & tree & native & 0.451 & 0.167 & 10.4 & 6601 \\
\hline Spondias purpurea $\mathrm{L}$. & ciriguela & dysentery & tree & exotic & 0.170 & 0.071 & 20.0 & n.c \\
\hline Spondias tuberosa Arr.Cam. & Imbu & stomachache & tree & native & 0.007 & 0.001 & 0.0 & 6604 \\
\hline Astronium fraxinifolium Schott. & gonçalave & flu & tree & native & 0.150 & 0.058 & 20.0 & 6690 \\
\hline \multicolumn{9}{|l|}{ ANNONACEAE } \\
\hline Annona squamosa $\mathrm{L}$. & pinha & fever & tree & exotic & 0.013 & 0.007 & 0.0 & 6600 \\
\hline Annona coriacea Mart. & ariticum & dysentery & shrub & native & 0.150 & 0.073 & 5.9 & 6614 \\
\hline \multicolumn{9}{|l|}{ APIACEAE } \\
\hline Apium sp. & milindro & high pressure & herb & & 0.020 & 0.011 & 0.0 & 6615 \\
\hline Coriandrum sativum $\mathrm{L}$. & coentro & child stomachache & herb & exotic & 0.013 & 0.003 & 0.0 & 6670 \\
\hline Foeniculum vulgare Mill. & endro & heartburn & herb & exotic & 0.183 & 0.046 & 18.8 & n.c \\
\hline Pimpinella anisum L. & erva doce & general pain & herb & exotic & 0.190 & 0.057 & 11.1 & 6606 \\
\hline \multicolumn{9}{|l|}{ APOCYNACEAE } \\
\hline Catharanthus roseus L. & boa noite & healing wounds or scars & herb & exotic & 0.007 & 0.004 & 0.0 & 6596 \\
\hline Hancornia speciosa Gomes & mangaba & varicose veins & tree & native & 0.601 & 0.235 & 75.0 & 6700 \\
\hline $\begin{array}{l}\text { Himatanthus drasticus (Mart.) } \\
\text { Plumel. }\end{array}$ & janaguba & gastritis & tree & native & 1.085 & 0.446 & 88.9 & 6660 \\
\hline Secondatia floribunda A. DC. & catuaba cipó & dilated veins & climber & native & 0.026 & 0.010 & 75.0 & n.c \\
\hline \multicolumn{9}{|l|}{ ARECACEAE } \\
\hline $\begin{array}{l}\text { Acrocomia aculeata (Jacq.) Lodd. } \\
\text { Ex Mart. }\end{array}$ & macaúba & to clean the intestine & tree & native & 0.013 & 0.002 & 50.0 & 6590 \\
\hline Attalea speciosa Mart. & babaçu & bruise & tree & native & 0.007 & 0.003 & 0.0 & n.c \\
\hline Cocos nucifera L. & côco & dehydration & tree & native & 0.026 & 0.010 & 50.0 & n.c \\
\hline Syagrus cearencis Noblik & catolé & hernia & tree & native & 0.013 & 0.010 & 0.0 & n.c \\
\hline \multicolumn{9}{|l|}{ ASTERACEAE } \\
\hline Acanthospermum hispidum DC. & espinho cigano & bronchitis & herb & exotic & 0.131 & 0.044 & 0.0 & n.c \\
\hline Acmella oleracea (L.) R.K. Jansen & agrião & cough & herb & native & 0.046 & 0.009 & 25.0 & n.c \\
\hline Agerantum conyzoides (L.) L. & mentrasto & vaginal hemorrhage & herb & exotic & 0.065 & 0.019 & 0.0 & n.c \\
\hline Artemisia vulgaris $\mathrm{L}$. & anador & headache & herb & exotic & 0.301 & 0.109 & 7.7 & 6656 \\
\hline Artemisia absinthium L. & lorma & indigestion & herb & exotic & 0.183 & 0.085 & 5.6 & 8108 \\
\hline Bidens pilosa $\mathrm{L}$. & carrapicho de agulha & woman inflammation & herb & native & 0.052 & 0.019 & 16.7 & 6591 \\
\hline Egletes viscosa (L.) Less. & macela & digestive gases & herb & native & 0.399 & 0.136 & 16.3 & 6603 \\
\hline Helianthus annuus L. & girassol & thrombosis & herb & exotic & 0.288 & 0.069 & 13.0 & 6665 \\
\hline Indet & rosamelia & cutaneous illness & herb & & 0.007 & 0.006 & 0.0 & 6653 \\
\hline Tanacetum vulgare L. & pruma & indigestion & herb & exotic & 0.085 & 0.034 & 0.0 & n.c \\
\hline Vernonia condensata Backer. & boldo de chile & heartburn & shrub & exotic & 0.033 & 0.013 & 0.0 & n.c \\
\hline
\end{tabular}


Table 1 Medicinal plants mentioned in the free lists and collected by the locals within the FLONA region (Horizonte community, NE Brazil) (Continued)

\section{BIGNONIACEAE}

Anemopaegma laeve DC.

Tabebuia sp.

BIXACEAE

Bixa orellana L.

\section{BORAGINACEAE}

Heliotropium indicum L.

BRASSICACEAE

Brassica rapa $\mathrm{L}$.

\section{BROMELIACEAE}

Ananas sativus Schult. \&

Schult. f.

\section{BURSERACEAE}

indet

\section{CACTACEAE}

Cereus jamacaru DC.

Harrisia adscendens (Gürke)

Britton \& Rose

Melocactus bahiensis (Britton \& Rose) Luetzelb

Opuntia ficus-indica (L.) Mill.

Pereskia grandifolia Haw.

\section{CANNABACEAE}

Cannabis sativa $\mathrm{L}$.

\section{CAPPARACEAE}

Cleome spinosa Jacq.

\section{CAPRIFOLIACEAE}

Sambucus australis Cham.

\& Schltdl.

\section{CARICACEAE}

Carica papaya L.

\section{CARYOCARACEAE}

Caryocar coriaceum Wittm.

\section{CELASTRACEAE}

Maytenus distichophylla Mart. CONVOLVULACEAE

Ipomoea batatas (L.) Poir.

Ipomoea asarifolia (Desr.)

Roem. \& Schult.

\section{COSTACEAE}

Costus spicatus (jacq.) Sw.

\section{CRASSULACEAE}

Bryophyllum pinnatum (Lam.) Oken

\section{CUCURBITACEAE}

Citrullus lanatus (Thunb.)

Matsum. \& Nakai manacá

podaico

rheumatism

all kind of pain

hematoma

teething in children

brain hemorrhage

flu

headache

menopause

menopause

magical protection

dysentery

flu

back pain

flu

teething in children

constipation

throat and lung pain

back pain

nervousness

batata doce

salsa

cana de macaco

kidney stone

malva da costa

itching

vaginal inflammation

climber native $0.013 \quad 0.005$

100.0

6669

tree

native $0.052 \quad 0.020$

20.0

n.c

$\begin{array}{llll}\text { shrub native } & 0.098 & 0.023 & 75.0\end{array}$

6698

herb

$\begin{array}{llll}\text { exotic } & 0.275 & 0.069 & 16.7\end{array}$

n.c

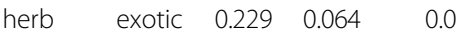

herb native $\quad 0.072 \quad 0.011 \quad 14.3$

6695

tree native $\quad 0.007 \quad 0.002$

6663

shrub native $0.007 \quad 0.002 \quad 0.0$

n.c

$\begin{array}{llll}\text { shrub native } & 0.020 & 0.008 & 33.3\end{array}$

6580

$\begin{array}{llll}\text { herb native } & 0.007 & 0.001 & 0.0\end{array}$

6618

$\begin{array}{lllll}\text { shrub exotic } & 0.013 & 0.005 & 0.0 & 6696\end{array}$

$\begin{array}{llll}\text { shrub native } & 0.020 & 0.009 & 0.0\end{array}$

n.c

shrub exotic $0.052 \quad 0.018 \quad 33.3$

6574

$\begin{array}{llll}\text { shrub exotic } & 0.039 & 0.016 & 16.7\end{array}$

6644

shrub

exotic $\quad 0.026 \quad 0.010$

0.0

n.c

$\begin{array}{lllll}\text { shrub exotic } & 0.098 & 0.030 & 9.1\end{array}$

6657

herb native $0.007 \quad 0.003 \quad 100.0$

6659

herb native $0.065 \quad 0.023 \quad 50.0$

6582

herb

exotic $\quad 0.680 \quad 0.270$

3.1

n.c

fever

herb

exotic $\quad 0.026 \quad 0.011$ 
Table 1 Medicinal plants mentioned in the free lists and collected by the locals within the FLONA region (Horizonte community, NE Brazil) (Continued)

\begin{tabular}{|c|c|c|c|c|c|c|c|c|}
\hline Cucurbita maxima Duch. ex Lam. & abóbora & malnutrition & herb & native & 0.007 & 0.004 & 0.0 & n.c \\
\hline Cucurbita argyrosperma Huber & gerimum & malnutrition & herb & exotic & 0.007 & 0.004 & 0.0 & n.c \\
\hline Luffa operculata (L.) Cogn. & cabacinho & abortive & climber & native & 0.013 & 0.008 & 50.0 & 8107 \\
\hline Momordica charantia L. & melão & itching & climber & exotic & 0.020 & 0.007 & 33.3 & 6577 \\
\hline Sechium edule (Jacq.) Sw. & chuchu & back pain & climber & exotic & 0.046 & 0.011 & 28.6 & n.c \\
\hline \multicolumn{9}{|l|}{ ERYTHROXYLACEAE } \\
\hline $\begin{array}{l}\text { Erythroxylum ampliofolium } \\
\text { (Mart.) O.E. Schulz }\end{array}$ & catuaba & erectile dysfunction & shrub & native & 0.222 & 0.093 & 80.0 & 6649 \\
\hline \multicolumn{9}{|l|}{ EUPHORBIACEAE } \\
\hline Croton blanchetianus Baill. & marmeleiro & diarrhea & shrub & native & 0.105 & 0.025 & 11.1 & 6594 \\
\hline Croton campestris A.St.-Hil. & velame & cutaneous illness & herb & native & 0.490 & 0.175 & 67.3 & 6622 \\
\hline Jatropha mollissima (Pohl) Baill. & pinhão bravo & headache & shrub & native & 0.046 & 0.016 & 0.0 & 6612 \\
\hline Jatropha gossypiifolia L. & pinhão roxo & neuralgia & shrub & native & 0.092 & 0.027 & 12.5 & 6645 \\
\hline Manihot esculenta Crantz & macaxeira & malnutrition & shrub & native & 0.007 & 0.003 & 0.0 & 8109 \\
\hline Manihot esculenta Crantz & mandioca & hematomas & shrub & native & 0.033 & 0.010 & 75.0 & 6691 \\
\hline Ricinus communis $\mathrm{L}$. & mamona & constipation & shrub & exotic & 0.144 & 0.033 & 42.9 & n.c \\
\hline \multicolumn{9}{|l|}{ FABACEAE } \\
\hline $\begin{array}{l}\text { Acosmium dasycarpum (Vogel.) } \\
\text { Yakovlev }\end{array}$ & pau pra tudo & all kind of pain & tree & native & 0.111 & 0.058 & 35.7 & n.c \\
\hline $\begin{array}{l}\text { Amburana cearensis (Allemão) } \\
\text { A.C. Sm. }\end{array}$ & imburana & bronchitis & tree & native & 0.314 & 0.076 & 8.3 & n.c \\
\hline Bauhinia outimouta Aubl. & mororó & teething in children & shrub & native & 0.085 & 0.023 & 22.2 & 6647 \\
\hline Bauhinia variegata $\mathrm{L}$. & pata de vaca & diabetes & tree & exotic & 0.059 & 0.023 & 16.7 & n.c \\
\hline Bowdichia virgilioides Kunth & sicupira & back pain & tree & native & 0.229 & 0.104 & 30.4 & n.c \\
\hline Caesalpinia ferrea C.Mart. & pau ferro & bronchitis & tree & native & 0.163 & 0.056 & 41.2 & n.c \\
\hline Cajanus cajan (L.) Millsp. & andu & hoarse & shrub & exotic & 0.118 & 0.030 & 8.3 & n.c \\
\hline Centrosema sp. & alcançu & bronchitis & shrub & native & 0.569 & 0.215 & 70.9 & 6699 \\
\hline Copaifera langsdorffii Desf. & podoia & arthritis & tree & native & 0.425 & 0.153 & 47.2 & 6661 \\
\hline Crotalaria incana L. & chucalinho & colic & shrub & native & 0.013 & 0.004 & 0.0 & n.c \\
\hline Dimorphandra gardneriana Tul. & faveira & back pain & tree & native & 0.059 & 0.093 & 100.0 & 6598 \\
\hline Dioclea grandiflora Benth. & mucunã & back pain & climber & native & 0.007 & 0.005 & 100.0 & n.c \\
\hline $\begin{array}{l}\text { Enterolobium contortisiliquum } \\
\text { (Vell.) Morong }\end{array}$ & tamburí & stomachache & tree & native & 0.052 & 0.020 & 0.0 & n.c \\
\hline $\begin{array}{l}\text { Hymenaea stignocarpa Mart. ex. } \\
\text { Hayne }\end{array}$ & jatobá & expectorant & tree & native & 0.634 & 0.274 & 55.9 & 6585 \\
\hline $\begin{array}{l}\text { Leucaena leucocephala (Lam.) } \\
\text { de Wit }\end{array}$ & linhaça & high cholesterol & tree & exotic & 0.105 & 0.024 & 55.6 & n.c \\
\hline Mimosa pudica $\mathrm{L}$. & malicia & tooth pain & shrub & native & 0.007 & 0.005 & & 6689 \\
\hline Parkia platycephala Benth. & visgueiro & healing wounds & tree & native & 0.020 & 0.008 & 0.0 & 6654 \\
\hline Senna occidentalis (L.) Link & manjerioba & abortive & shrub & native & 0.072 & 0.029 & 0.0 & 6635 \\
\hline $\begin{array}{l}\text { Stryphnodendron rotundifolium } \\
\text { Mart. }\end{array}$ & barbatenã & healing wounds or scars & tree & native & 1.144 & 0.451 & 75.7 & n.c \\
\hline Vicia faba $\mathrm{L}$. & fava & carbuncle & climber & exotic & 0.007 & 0.001 & 100.0 & 6693 \\
\hline Vigna unguiculata L., Walp. & feijão de corda & carbuncle & climber & exotic & 0.026 & 0.002 & 66.7 & 6579 \\
\hline \multicolumn{9}{|l|}{ KRAMERIACEAE } \\
\hline Krameria tomentosa A. St.-Hil. & carrapicho de boi & abortive & shrub & native & 0.137 & 0.036 & 50.0 & n.c \\
\hline
\end{tabular}


Table 1 Medicinal plants mentioned in the free lists and collected by the locals within the FLONA region (Horizonte community, NE Brazil) (Continued)

\begin{tabular}{|c|c|c|c|c|c|c|c|c|}
\hline \multicolumn{9}{|l|}{ LAMIACEAE } \\
\hline Aeollanthus sp. & hortelã & throat pain & herb & exotic & & & & n.c \\
\hline Hyptis martiusii Benth. & cidreira brava & flu & shrub & native & 0.007 & 0.001 & 0.0 & n.c \\
\hline Mentha piperita L. & hortelã & throat pain & herb & exotic & 0.915 & 0.397 & 8.2 & 6607 \\
\hline Ocimum gratissimum L. & alfavaca & sinusitis & herb & exotic & 0.209 & 0.070 & 4.8 & 6605 \\
\hline Ocimum americanum L. & manjericão & inner ear pain & herb & exotic & 0.144 & 0.044 & 15.4 & n.c \\
\hline Ocimum basilicum L. & manjericão & inner ear pain & herb & exotic & & & & n.c \\
\hline Plectranthus neochilus Schltr. & boldo & indigestion & herb & exotic & 0.183 & 0.084 & 0.0 & 6621 \\
\hline $\begin{array}{l}\text { Plectranthus amboinicus (Lour.) } \\
\text { Spreng. }\end{array}$ & malva do reino & expectorant & herb & exotic & 1.000 & 0.448 & 7.5 & n.c \\
\hline Plectranthus barbatus Andrews & sete dor & indigestion & shrub & exotic & 0.170 & 0.072 & 5.3 & 6640 \\
\hline $\begin{array}{l}\text { Rhaphiodon echinus } \\
\text { (Nees \& Mart.) Schauer }\end{array}$ & betonca & pain when urinate & herb & native & 0.052 & 0.008 & 0.0 & 6692 \\
\hline Rosmarinus officinalis L. & alecrim & stomachache & herb & exotic & 0.967 & 0.438 & 33.0 & 6597 \\
\hline \multicolumn{9}{|l|}{ LAURACEAE } \\
\hline Persea americana Mill. & abacate & diuretic & tree & exotic & 0.229 & 0.060 & 14.3 & 6595 \\
\hline \multicolumn{9}{|l|}{ LYTHRACEAE } \\
\hline Punica granatum L. & romã & throat inflammation & shrub & exotic & 0.235 & 0.089 & 51.7 & 6611 \\
\hline \multicolumn{9}{|l|}{ MALPHIGIACEAE } \\
\hline Byrsonima sericea DC. & murici & hemorrhage & tree & native & 0.052 & 0.015 & 16.7 & n.c \\
\hline Malphigia glabra L. & acerola & anemia & shrub & exotic & 0.046 & 0.015 & 66.7 & 6688 \\
\hline Stigmaphyllon paralias A. Juss. & salsa parrilha & scabies & shrub & native & 0.039 & 0.015 & 66.7 & n.c \\
\hline \multicolumn{9}{|l|}{ MALVACEAE } \\
\hline $\begin{array}{l}\text { Abelmoschus esculentus } \\
\text { L. Moench }\end{array}$ & quiabo & snake bite & herb & exotic & 0.007 & 0.006 & 0.0 & 6572 \\
\hline Gossypium barbadense L. & algodão & carbuncle & shrub & exotic & 0.026 & 0.004 & 0.0 & n.c \\
\hline Gossypium hirsutum L. & algodão & carbuncle & shrub & exotic & & & & n.c \\
\hline Sida cordifolia L. & malva branca & teething in children & herb & native & 0.216 & 0.068 & 26.3 & n.c \\
\hline Waltheria indica $\mathrm{L}$. & malva & "no local use" & herb & native & 0.000 & 0.005 & 100.0 & n.c \\
\hline \multicolumn{9}{|l|}{ MELIACEAE } \\
\hline Azadirachta indica A. Juss. & neem & lice & tree & exotic & 0.020 & 0.007 & 0.0 & 6602 \\
\hline \multicolumn{9}{|l|}{ MENISPERMACEAE } \\
\hline Cissampelos ovalifolia DC. & orelha de onça & indigestion & herb & native & 0.320 & 0.109 & 32.3 & 6641 \\
\hline \multicolumn{9}{|l|}{ MUSACEAE } \\
\hline Musa paradisiaca L. & banana & flu & herb & exotic & 0.033 & 0.011 & 0.0 & 6620 \\
\hline \multicolumn{9}{|l|}{ MYRTACEAE } \\
\hline $\begin{array}{l}\text { Campomanesia eugenioides } \\
\text { (Cambess.) D.Legrand }\end{array}$ & fruta bola & malnutrition & shrub & native & 0.013 & 0.001 & 50.0 & n.c \\
\hline Eucalyptus citriodora F. Muell. & eucalipto & sinusitis & tree & exotic & 0.673 & 0.220 & 26.6 & 6576 \\
\hline Eugenia uniflora L. & pitanga & intestinal parasites & shrub & native & 0.065 & 0.016 & 0.0 & n.c \\
\hline Myrciaria sp. & cambuí & $\begin{array}{l}\text { the informant do not } \\
\text { record the local use }\end{array}$ & shrub & native & 0.000 & 0.002 & 0.0 & 6650 \\
\hline Psidium guajava L. & goiaba & healing wounds & tree & native & 0.065 & 0.025 & 12.5 & n.c \\
\hline \multirow[t]{2}{*}{ Psidium guajava L. } & goiaba branca & dysentery & tree & native & 0.078 & 0.027 & 12.5 & n.c \\
\hline & goiabinha & dysentery & shrub & native & 0.065 & 0.033 & 0.0 & 6664 \\
\hline
\end{tabular}


Table 1 Medicinal plants mentioned in the free lists and collected by the locals within the FLONA region (Horizonte community, NE Brazil) (Continued)

\begin{tabular}{|c|c|c|c|c|c|c|c|c|}
\hline \multicolumn{9}{|l|}{$\begin{array}{l}\text { Psidium sobraleanum Proença } \\
\text { \& Landrum }\end{array}$} \\
\hline Psidum myrsinites DC. & araçá vermelho & diarrhea & tree & native & 0.190 & 0.088 & 4.3 & 6619 \\
\hline Syzygium cumini (L.) Skeels & azeitona preta & diabetes & tree & exotic & 0.013 & 0.001 & 0.0 & 6610 \\
\hline \multicolumn{9}{|l|}{ NYCTAGINACEAE } \\
\hline Boerhavia diffusa L. & pega pinto & skin diseases in children & herb & exotic & 0.059 & 0.014 & 0.0 & n.c \\
\hline Mirabilis jalapa L. & bonina & brain hemorrhage & herb & exotic & 0.033 & 0.009 & 0.0 & 6588 \\
\hline \multicolumn{9}{|l|}{ OLACACEAE } \\
\hline Ximenia americana $\mathrm{L}$. & ameixa & healing wounds or scars & shrub & native & 0.621 & 0.233 & 46.7 & 6571 \\
\hline \multicolumn{9}{|l|}{ PASSIFLORACEAE } \\
\hline Passiflora edulis Sims & maracujá & insomnia & climber & native & 0.085 & 0.026 & 36.4 & 8104 \\
\hline Passiflora cincinnata Mast. & $\begin{array}{l}\text { maracujá do } \\
\text { mato }\end{array}$ & insomnia & climber & native & 0.190 & 0.047 & 5.0 & 6581 \\
\hline Passiflora sp. & maracujá peroba & calmative & climber & native & 0.007 & 0.002 & 100.0 & 6648 \\
\hline Turnera subulata Sm. & xanana & renal infection & herb & native & 0.092 & 0.030 & 33.3 & 6609 \\
\hline \multicolumn{9}{|l|}{ PHYLLANTHACEAE } \\
\hline Phyllanthus urinaria $\mathrm{L}$. & quebra pedra & kidney stone & herb & native & 0.170 & 0.055 & 10.5 & n.c \\
\hline \multicolumn{9}{|l|}{ PHYTHOLACCACEAE } \\
\hline Petiveria alliacea L. & tipí & inner ear pain & herb & native & 0.052 & 0.010 & 0.0 & 6658 \\
\hline \multicolumn{9}{|l|}{ PLANTAGINACEAE } \\
\hline Plantago sp. & chapeu de couro & $\begin{array}{l}\text { the informant do } \\
\text { not record the local use }\end{array}$ & herb & & 0.000 & 0.004 & 0.0 & 6638 \\
\hline \multicolumn{9}{|l|}{ POACEAE } \\
\hline $\begin{array}{l}\text { Brachiaria plantaginea (Link) } \\
\text { Hitchc. }\end{array}$ & capim de planta & diuretic & herb & exotic & 0.026 & 0.004 & 0.0 & n.c \\
\hline $\begin{array}{l}\text { Cymbopogon citratus (DC) } \\
\text { Stapf. }\end{array}$ & capim santo & calmative & herb & exotic & 0.444 & 0.191 & 5.8 & 6628 \\
\hline $\begin{array}{l}\text { Cymbopogon winterianus } \\
\text { Jowitt ex Bor }\end{array}$ & citronela & sinusitis & herb & exotic & 0.020 & 0.005 & 0.0 & 6586 \\
\hline Saccharum officinarum L. & cana & calmative & herb & exotic & 0.131 & 0.039 & 0.0 & 6568 \\
\hline Zea mays $L$. & milho & urinary infection & herb & exotic & 0.013 & 0.001 & 0.0 & 6646 \\
\hline \multicolumn{9}{|l|}{ POLYGALACEAE } \\
\hline Polygala paniculata $\mathrm{L}$. & caninana & rheumatism & herb & native & 0.046 & 0.023 & 66.7 & 6642 \\
\hline \multicolumn{9}{|l|}{ PROTEACEAE } \\
\hline Roupala montana Aubl. & congonha & liver problems & tree & native & 0.124 & 0.039 & 40.0 & 6666 \\
\hline \multicolumn{9}{|l|}{ RHAMNACEAE } \\
\hline Ziziphus joazeiro Mart. & juazeiro & expectorant & tree & native & 0.092 & 0.021 & 20.0 & n.c \\
\hline \multicolumn{9}{|l|}{ RUBIACEAE } \\
\hline Coffea arabica $\mathrm{L}$. & café & throat pain & shrub & exotic & 0.052 & 0.023 & 0.0 & n.c \\
\hline $\begin{array}{l}\text { Tocoyena formosa (Cham. } \\
\text { \& Schltdl.) K.Schum. }\end{array}$ & genipapim & bone fracture & shrub & native & 0.013 & 0.010 & 33.3 & n.c \\
\hline \multicolumn{9}{|l|}{ RUTACEAE } \\
\hline Citrus sinensis (L.) Osbeck & laranja & somniferous and calmative & tree & exotic & 0.340 & 0.107 & 17.1 & 6651 \\
\hline Citrus aurantium (L.) & laranja da terra & intestinal parasites & tree & exotic & 0.020 & 0.009 & 25.0 & 6701 \\
\hline Citrus limon (L.) Burm. f. & limão & cold & tree & exotic & 0.163 & 0.052 & 36.4 & n.c \\
\hline \multirow[t]{2}{*}{ Murraya paniculata (L.) Jack } & jasmim laranja & indigestion & shrub & exotic & 0.124 & 0.045 & 0.0 & n.c \\
\hline & jaborandi & fever & shrub & native & 0.046 & 0.019 & 11.1 & 6573 \\
\hline
\end{tabular}


Table 1 Medicinal plants mentioned in the free lists and collected by the locals within the FLONA region (Horizonte community, NE Brazil) (Continued) Pilocarpus microphyllus Stapf
ex Wardleworth

Ruta graveolens L.

SALICACEAE

Casearia javitensis Kunth

\section{SANTALACEAE}

Phoradendron mucronatum (DC.) Krug \& Urb.

\section{SAPINDACEAE}

Magonia pubescens A. St.-Hil.

tingui

Serjania sp.

Talisia escuelenta A. St.-Hil.)

Radlk.

arruda

café bravo

estreico de passarinho measles

general pain

fatigue

shro

herb

exotic $\quad 1.183 \quad 0.479$

59.4

6643

cipó de vaqueiro

healing wounds

pitomba

hematoma

rheumatism

\section{SAPOTACEAE}

Pouteria glomerata (Miq.)

Radlk.

mamelada

cough

vassourinha

teething in children

cough

Smilax campestris Griseb.

japecanga

(a)

\section{SOLANACEAE}

Capsicum frutescens $\mathrm{L}$.

Solanum erianthum D. Don

Solanum sp.

Solanum agrarium Sendtn.

Solanum americanum Mill.

\section{URTICACEAE}

Pilea microphylla (L.) Liebm.

\section{VERBENACEAE}

Lantana camara L.

Lippia gracilis Schauer

Lippia alba Mill.) N.E.Br.

ex Britton \& P.Wilson

Lippia alba Mill.) N.E.Br.

ex Britton \& P.Wilson

\section{VIOLACEAE}

Hybanthus calceolaria (L.) Oken.

pimenta ardosa

jurubeba

cutaneous illness

jurubeba

skin irritation

melancia da praia

skin irritation

menopaue

vamora

menopause

zezinho

breathing difficulties

chumbim

alecrim do mato

carmelitana

vaginal hemorrhage

carbuncle

intestinal problems

cidreira

non appetite

papaconha

teething in children

herb

native

$0.346 \quad 0.101$

32.3

6652

\section{VOCHYSIACEAE}

Qualea parviflora Mart.

pau piranha

for the placenta to came tre out after birth

babosa

gastritis

low blood pressure

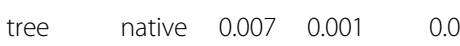

climber native $\quad 0.007 \quad 0.004 \quad 0.0$

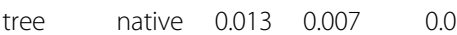

6575

6593

6629

\section{XANTHORRHOEACEAE}

Aloe vera (L.) Burm.f.

ZINGIBERACEAE

Alpinia zerumbet (Pers.) B.L.Burtt colônia \& R.M.Sm 
the herbaceous ones, dominate the pharmacopeia in the Brazilian Atlantic Forest.

A total of $64(38.8 \%)$ exotic and $101(61.2 \%)$ native ethnospecies were reported among the medicinal plants collected within the FLONA region (the origin could not be determined for three ethnospecies due to taxonomic difficulties). The exotic plants were divided into 34 herbs (53.1\%), 14 shrubs (21.9\%), 12 trees $(18.7 \%)$, and four climbers $(6.3 \%)$. When the exotic plants were excluded from the analysis of the native pharmacopoeia, 34 trees (33.7\%), 32 shrubs (31.7\%), 25 herbs (24.8\%), nine climbers $(8.9 \%)$, and one parasite $(0.9 \%)$ were recorded, indicating that the dominance of herbaceous plants in this pharmacopeia is an effect of the exotic medicinal flora (Figure 1). With the removal of the exotic plants, the difference between the percentages of woody and non-woody plants in the pharmacopoeia increased, resulting in 30 non-woody (29.7\%) and 71 woody species (70.3\%). In the case of the native pharmacopoeia, the woody plants (mostly of arboreal habit) dominated, a finding that was also reported for the Caatinga [26] and does not match the predictions made based on the apparency hypothesis from the perspective of biochemical fundamentals. Thus, the influx of medicinal species from other continents than South America has an effect on the proportion of habits of the plants in the local pharmacopeia and, therefore, on interpreting this pharmacopoeia from the perspective of the apparency hypothesis.

Although outside of the scope of this study, it is important to mention that the apparency hypothesis has other approximations. Phillips and Gentry [27] proposed that, from an ecological perspective, apparent (conspicuous) plants are the more abundant ones in the vegetation and therefore subject to be experienced more frequently by human communities, which will lead to a major development of uses for those. Based on this idea,

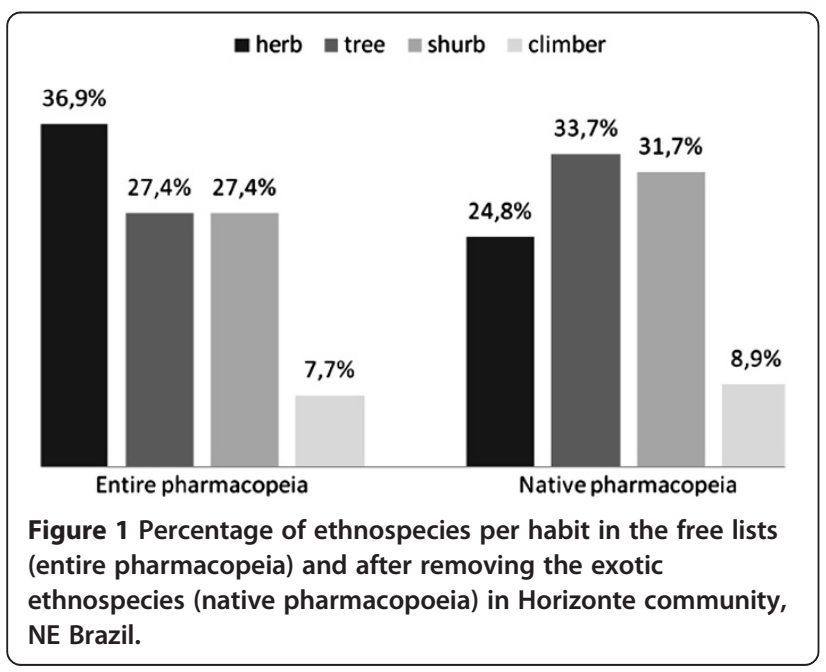

one could suppose that if a plant species is widespread and abundant in a certain environment it is apparent regardless of its habit but this assumption has not yet been tested.

\section{Habits of ethnospecies and their practical values}

A total of 90 ethnospecies were reportedly used during the month preceding the day of the interview (i.e., were recently used). Of these ethnospecies, 11 were from other regions and 79 were collected within the FLONA region; the latter can be divided into the following groups: 37 herbs (46.8\%), 21 trees (26.6\%), 17 shrubs (21.5\%), 3 climbers (3.8\%), and one plant of indeterminate habit (one plant not collected). Again, the herbaceous habit was characteristic of most of the ethnospecies. The proportion of herbs increased in the recently used plants while the other habits decreased compared to the percentages of plants per habit in the free lists, suggesting that herbaceous plants were the habit of most practical importance.

Supporting these results, among the plants used recently, 40 were non-woody $(50.6 \%)$ and 38 were woody species $(48.1 \%)$, increasing the percentage of non-woody plants to the point of surpassing the percentage of woody plants when compared to the values observed in the free lists. These results, obtained via both means of grouping the plants to test the apparency hypothesis, suggest that non-woody plants, specifically those of herbaceous habit, have the most practical value. This finding strongly indicates that these plants, characterized from the biochemical perspective of the apparency hypothesis as having highly bioactive compounds content, are the most desirable for treating diseases within the Horizonte community.

The results obtained for the plants used during the month preceding the interviews are influenced by various factors that may skew our interpretation. The period of the year when the interviews were conducted corresponded to the region's rainy season, concentrated from December to April [18], a period in which one would expect the herbaceous component of the vegetation to be fully available in the environment, and that may have led to greater use of plants with that habit. These results may also reflect the pattern of diseases experienced by Horizonte inhabitants during this period (e.g., a cold virus spread throughout the population), leading to the use of certain plants.

Exotic plants corresponded to 50\% (39 ethnospecies) of the medicinal plants used during the month preceding the interview, having a higher representation than in the entire pharmacopeia (38.8\%). When the exotic species were removed from the group of plants mentioned as being used within the last month, the percentage of ethnospecies per habit changed, highlighting the following 
distribution among the native plants used: 14 ethnospecies each corresponded to trees (35.9\%) and to herbs (35.9\%), followed by 9 shrubs (23\%) and two climbers (5.1\%). The exotic medicinal flora highlights the herbaceous habit, this time on the practical level because when the exotic plants are removed, trees and herbs formed the highest percentages of ethnospecies richness among those recently used.

\section{Collection sites}

The results of the informant consensus associating ethnospecies to collection sites are shown in Table 2. In this analysis, the term "other regions" was considered to refer to collection sites that, although not sites in the sense of areas, are still sources of medicines for Horizonte residents; including this term allows for comparisons with this group of plants.

The ethnospecies richness cited in the free lists exhibited significant differences depending on the collection sites $\left(\mathrm{X}^{2} 16.40 ; 4 \mathrm{df}\right.$ [degrees of freedom]; $\left.\mathrm{p}<0.01\right)$, which corresponded to differences between anthropogenic areas and native vegetation $\left(\mathrm{X}^{2}=10.39 ; 2 \mathrm{df} ; \mathrm{p}<0.01\right)$, between anthropogenic areas and other regions $\left(X^{2}=6.05 ; 2 \mathrm{df}\right.$; $\mathrm{p}<0.05)$, and between native vegetation and other regions $\left(\mathrm{X}^{2}=6.50 ; 2 \mathrm{df} ; \mathrm{p}<0.05\right)$.

The anthropogenic areas differed significantly from other regions in being the site with the highest number of ethnospecies cited and were the principal source (collecting site) of medicinal plants for the Horizonte inhabitants. This result reinforces the findings of other authors that secondary vegetation or the vegetation of anthropogenic areas is the main source of medicinal plants in the pharmacopoeia of human communities in tropical forests [7,28-30].

Based on the informant consensus, $72.6 \%$ (45 ethnospecies) of all the plants, $34.8 \%$ (16) of the trees, $50 \%$ (23)

Table 2 Medicinal ethnospecies richness per collection site with two or more informants and $\mathbf{7 5 \%}$ or more citations for the site, with two or more informants and less than $75 \%$ citations for the site, and with only one informant (Horizonte community, NE Brazil)

\begin{tabular}{|c|c|c|c|c|c|c|c|}
\hline \multirow{4}{*}{$\begin{array}{l}\text { Ethnospecies } \\
\text { richness }\end{array}$} & & \multicolumn{6}{|c|}{ Collection sites } \\
\hline & & \multicolumn{2}{|c|}{$\begin{array}{l}\text { Anthropogenic } \\
\text { areas }\end{array}$} & \multicolumn{2}{|c|}{$\begin{array}{l}\text { Native } \\
\text { vegetation }\end{array}$} & \multicolumn{2}{|c|}{$\begin{array}{l}\text { Other } \\
\text { regions }\end{array}$} \\
\hline & \multirow[b]{2}{*}{$\begin{array}{l}\geq 75 \% \\
\text { citations }\end{array}$} & $\mathrm{FL}$ & $\mathrm{RU}$ & $\mathrm{FL}$ & $\mathrm{RU}$ & $\mathrm{FL}$ & $\mathrm{RU}$ \\
\hline & & 90 & 54 & 27 & 13 & 26 & 10 \\
\hline & $<75 \%$ citations & 57 & 29 & 42 & 26 & 28 & 15 \\
\hline & $\begin{array}{l}\text { Only one } \\
\text { informant }\end{array}$ & 26 & 1 & 8 & 0 & 18 & 1 \\
\hline & Total & 173 & 84 & 77 & 39 & 72 & 26 \\
\hline
\end{tabular}

The analysis was based on the free lists (FL) and lists of plants recently used (RU). Horizonte community, NE Brazil. of the shrubs, and $38.5 \%$ (5) of the climbers were strongly associated with collection in anthropogenic areas. When the plants were categorized as woody and non-woody, $70.4 \%$ (50) of the non-woody and $40.2 \%$ (39) of the woody plants were strongly associated with collection in anthropogenic areas. Additionally, 79.7\% (51) of exotic plants were strongly associated with these areas. These results indicate a close relationship between anthropogenic areas and the collection of non-woody medicinal plants, mostly those with an herbaceous habit, as well as of exotic plants.

The greater value associated with anthropogenic areas was an expected result from the perspective of the biochemical fundamentals of the apparency hypothesis because anthropogenic areas, in our classification, are open and cultivated areas as well as areas with vegetation in the early stages of plant succession. Therefore, these regions were considered to be areas where non-apparent species dominate, a fact that was corroborated by the high incidence of reports of collection of non-woody herbaceous plants. The greater presence of woody plants, primarily shrubs, in anthropogenic areas certainly helps in differentiating these regions as the main source of medicinal plants and contributes to the higher importance attributed to these areas. Among the woody plants strongly associated with collection in anthropogenic areas, exotic species corresponded to $52.2 \%$ of the shrubs and $62.5 \%$ of the trees, indicating that the contribution of woody plants to the higher importance of anthropogenic areas as medicinal collection sites was, in more than half of the cases, due to the contribution of exotic plants.

Explanations of both an environmental and a cultural nature may contribute to understanding the role of anthropogenic areas and native vegetation in tropical pharmacopoeias. In the Brazilian semi-arid lands within the Caatinga ecosystem, native vegetation zones are the primary source of medicinal plants that are effectively used [15]. This pattern makes sense because in seasonal climates such as that of the Caatinga, where annual rainfall is irregularly distributed throughout the year and ranges between 250 and 1,200 $\mathrm{mm}$, plant use is directed toward the perennial parts of woody plants (bark and roots) that are available throughout the year, in contrast to the herbaceous plants that have limited availability during the dry season [6]. Thus, there is a preference for using medicinal woody plants sought primarily in areas of native vegetation $[17,31]$.

Explanations of a cultural nature may also contribute to understanding the dominance of herbs and anthropogenic areas in tropical forest pharmacopoeias. For example, in the Brazilian Atlantic Forest, where the annual rainfall is evenly distributed and varies between 1,800 and 2,100 $\mathrm{mm}$, the limited contact that slaves historically had with the region"s primary vegetation (due to their 
captivity and work in cultivated areas coupled with a high rate of deforestation in this ecosystem) was a factor reported by Voeks [12] to explain why anthropogenic areas and herbaceous plants are important in the current local pharmacopeia. One must also consider the influence of other cultural archetypes that have been important in forming the local pharmacopoeia over time, of which knowledge and practices are also present in the current local pharmacopeia to varying extents [32]. In this regard, the presence of the dominant European discourse, which attempted to impose its lifestyles on the inhabitants of the "new lands", is also noteworthy [33]. Many European medicines were incorporated into the African-Brazilian pharmacopoeia, which were either cultivated or found in local secondary vegetation [12].

However, following the line of explanations from the environmental standpoint, it is expected that in Atlantic Forest regions, due to the lack of a dry season [34], herbs and anthropogenic areas became prominent as the main sources of medicinal plants. The 'Cerrado' biome, with annual rainfall between 800 and 2,000 mm and a marked dry period [35], is intermediate between the Caatinga and Atlantic Forest biomes in terms of annual rainfall. The results obtained regarding the greater prominence of herbaceous plants in terms of ethnospecies richness and practical importance are similar to those found by other authors in tropical rainforests [12,29].

The native vegetation is primarily a source of medicinal woody flora. Of the 27 native plants that are strongly associated with collection in the native vegetation, 96.1\% (25 ethnospecies) are native woody species. Upon separating the native vegetation between outside and inside the FLONA to evaluate the conservation unit's role as a site for gathering medicinal plants, there were no significant differences in the ethnospecies richness of the plants that were cited as collected at these two sites ( $\mathrm{G}$ test $5.44 ; 2 \mathrm{df} ; \mathrm{p}>0.05$ ). This result indicates that the Horizonte residents find medicinal plants in the native vegetation that can be collected both outside and within the FLONA.

The total values in Table 2 indicate the number of ethnospecies with at least one citation for each collection site; these values are expressed in percentages in Figure 2.

The importance of anthropogenic areas as sites for collecting medicinal plants is even more apparent when analyzing the plants that were recently used (Figure 2). This information about the collection site, although recorded using interviews, corroborates the results obtained by Gavin [8], who directly recorded the collection sites of plants used in the Peruvian Amazon. He reported more collection of medicinal plants in secondary vegetation that had developed after cultivated land was

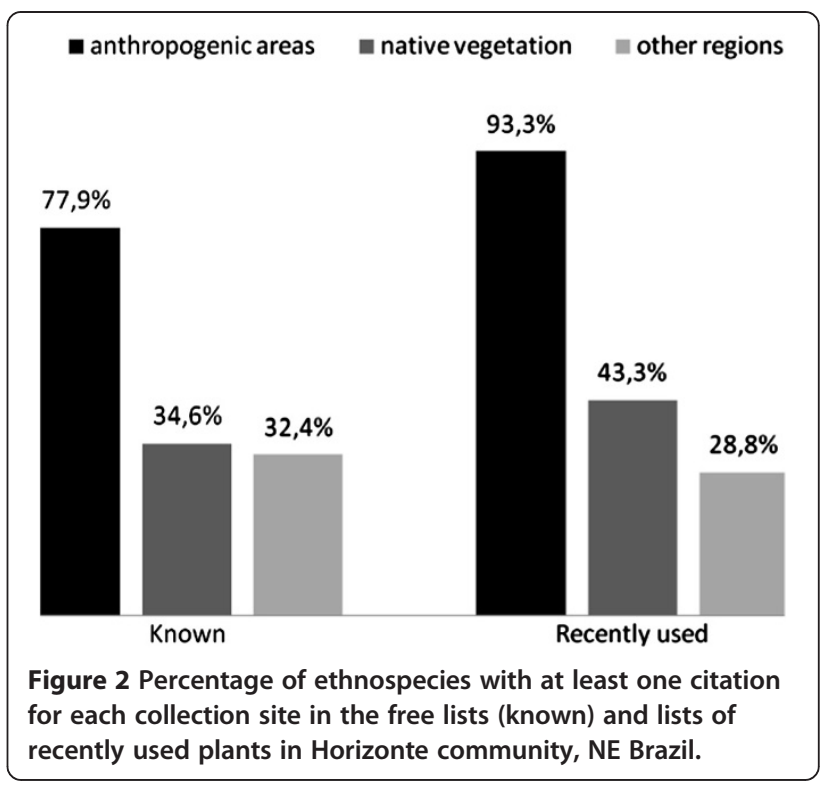

abandoned than in primary forest. Similar percentages of plants collected in anthropogenic areas (between $74 \%$ and $80 \%$ ) were obtained by Frei et al. [36] in their study with the Mixe and Zapotec indigenous groups in Mexico.

The percentage of medicinal plants from other regions was lower in the group of recently used plants compared with the known plants (Figure 2). This finding may be an effect of the accessibility of these resources because the plants from other regions must be purchased commercially (involving monetary costs) or acquired during travel to surrounding Caatinga sites. In contrast, the percentage of native vegetation plants was higher among the plants recently used than for the known plants, indicating that the native vegetation was more important in terms of the practical level or actual use of medicinal plants.

\section{Cognitive aspects of use}

Using the information from the free lists, a strong correlation between salience and use value was found ( $\mathrm{rs}=0.950$; $\mathrm{p}<0.001$ ), i.e., plants with a high use potential are listed by more people and are ranked higher within the lists, or in other words, people quickly remember plants with a high use potential or versatility.

Herbs and trees exhibited no significant differences in use values $(H=0.0111, p>0.05)$, and these groups did not differ significantly from shrubs $(\mathrm{H}=3.3941$ and $\mathrm{H}=$ 2.4536, respectively, $\mathrm{p}>0.05$ ). Herbs, trees, and shrubs had use values significantly different from that of climbers $(\mathrm{H}=7.0096, \mathrm{H}=6.3171$, and $\mathrm{H}=3.9326$, respectively, $\mathrm{p}<0.05)$. Trees had, on average, higher use values $(0.205 \pm 0.283)$, followed by herbs $(0.201 \pm 0.267)$, shrubs $(0.098 \pm 0.143)$, and climbers $(0.042 \pm 0.054)$. However, the use value for plants is distributed independently from 
lignification because the use values for woody plants did not significantly differ from those of non-woody plants $(\mathrm{H}=0.5186 \mathrm{p}>0.05)$.

According to the apparency hypothesis based on biochemical fundamentals, non-woody plants and herbs were expected to have higher use values and therefore higher versatility, but the results indicate that medicinal trees, shrubs, and herbs are equally versatile, and woody plants are as versatile as non-woody ones.

Among the native medicinal plants used by the rural communities that inhabit Caatinga areas, the trees and shrubs exhibited higher occurrences of quantitative compounds (phenols and tannins) and qualitative compounds (alkaloids, triterpenes, and quinones) [26]. These results partially meet the predictions made based on the biochemical fundamentals of the apparency hypothesis, which may, for example, help explain the versatility of apparent plants in the studied pharmacopoeia. In the same study, Almeida et al. [26] found that medicinal trees and shrubs were the habits with higher relative importance, above herbs, which are similar results to those obtained in the present study, indicating the possibility of a similarity in this respect between the Cerrado and Caatinga pharmacopoeias. Contrasting results were obtained in rainforests such as the Bolivian Amazon, where herbs have, on average, a higher medicinal use value than all the other habits (trees, shrubs, and climbers) [30].

Use value was also not influenced by origin; there were no differences between native and exotic plants $(\mathrm{H}=$ $3.2457 \mathrm{p}>0.05$ ), indicating that the Horizonte residents have exploited as many medicinal uses for exotic plants as for native medicinal species. According to the diversification hypothesis, this result may indicate that exotic plants have been included within the pharmacopeia to treat specific diseases because they contain biochemical compounds that do not exist in the local pharmacopoeia or have higher concentrations of those already present [37]. Cultural and biochemical analyses would have to be conducted to delve into the cognitive aspects of the use of exotic plants within this pharmacopoeia.

\section{Ethnospecies habit and commercial value}

Nine medicinal ethnospecies, all of which were native, were associated with more than $75 \%$ affirmative citations for commercial value; of these ethnospecies, 8 were woody ( 5 trees and 3 shrubs), and one was a non-woody (herb) plant. Again separating the native vegetation outside and within the FLONA, it was found that for five of the ethnospecies, $75 \%$ or more of the citations were for collection within the FLONA, three were exclusively from anthropogenic areas $(100 \%$ of the citations were for this collection site), and one had less than $75 \%$ of its citations for any particular collection site (Table 3).

It was expected that non-apparent plants, non-woody plants or plants with an herbaceous habit, characterized by having highly bioactive compounds, would have more ethnospecies with high percentages of affirmative citations for commercial value. However, contrary to expectations, the woody trees had a higher number of commercially important ethnospecies. Of the 12 plant species most commonly sold in the markets in the city of Belem in the Brazilian Amazon, nine are woody natives (six trees, two shrubs, and one climber) and the remaining three are

\begin{tabular}{|c|c|c|c|c|c|}
\hline Ethnospecies/species & Habit & Collection sites & Part sold & $\begin{array}{l}\text { Commercial } \\
\text { value }\end{array}$ & $\begin{array}{l}\text { No. of } \\
\text { informants }\end{array}$ \\
\hline $\begin{array}{l}\text { "Fava d"anta' tree/Dimorphandra } \\
\text { gardneriana Tul. }\end{array}$ & Tree & $\begin{array}{l}52.7 \% \text { FLONA, } 18.2 \% \text { nat. veg. outside FLONA, } \\
29.1 \% \text { anthropogenic area }\end{array}$ & Fruit & $100 \%$ & 29 \\
\hline Malva/Waltheria indica $\mathrm{L}$. & Herb & $100 \%$ anthropogenic area & Inflorescence & $100 \%$ & 2 \\
\hline 'Pequi'/Caryocar coriaceum Wittm. & Tree & $\begin{array}{l}\text { 76.3\% FLONA, } 5.3 \% \text { native vegetation outside } \\
\text { FLONA, } 18.4 \% \text { anthropogenic area }\end{array}$ & $\begin{array}{l}{ }^{*} \text { Oil from fruit and } \\
\text { seed }\end{array}$ & $98.28 \%$ & 58 \\
\hline $\begin{array}{l}\text { 'Janaguba'/Himatanthus drasticus (Mart.) } \\
\text { Plumel. }\end{array}$ & Tree & $\begin{array}{l}\text { 85.6\% FLONA, } 11.0 \% \text { native vegetation outside } \\
\text { FLONA, 3.4\% anthropogenic area }\end{array}$ & Latex, stem bark & $88.89 \%$ & 99 \\
\hline $\begin{array}{l}\text { 'Catuaba'/Erythroxylum ampliofolium (Mart.) } \\
\text { O.E. Schulz }\end{array}$ & Shrub & $\begin{array}{l}\text { 81.8\% FLONA, } 12.1 \% \text { native vegetation outside } \\
\text { FLONA, 3.0\% anthropogenic area }\end{array}$ & Stem bark & $80 \%$ & 30 \\
\hline $\begin{array}{l}\text { 'Barbatimão'/Stryphnodendron rotundifolium } \\
\text { Mart.. }\end{array}$ & Tree & $\begin{array}{l}90.7 \% \text { FLONA, } 7.6 \% \text { native vegetation outside } \\
\text { FLONA, 1.7\%. anthropogenic area }\end{array}$ & Stem bark & $75.70 \%$ & 107 \\
\hline Cassava/Manihot esculenta Crantz & Shrub & $100 \%$ anthropogenic area & *Gum & $75 \%$ & 4 \\
\hline 'Mangaba'/Hancornia speciosa Gomes & Tree & $\begin{array}{l}\text { 87.5\% FLONA, 9.4\% native vegetation outside } \\
\text { FLONA, 3.1\% anthropogenic area }\end{array}$ & Latex, stem bark & $75 \%$ & 56 \\
\hline 'Urucum'/Bixa orellana L. & Shrub & $100 \%$ anth anthropogenic area & Seed & $75 \%$ & 8 \\
\hline
\end{tabular}


herbaceous exotic plants (extracontinental origin) [38]. In the Caatinga region, medicinal plant use is usually associated with removing the bark from tree species [17], and the bark from native trees is usually sold in popular markets [39]. These studies indicate that in other ecosystems, trees also constitute the habit of greater economic importance and are mostly native species, such as the trees shown in Table 3. The economic importance of native trees in different ecosystems may be due to cultural factors, such as the perception that naturally growing plants have more healing power [36], and the biochemical diversity of these plants, as in the case of Caatinga [26]. These factors together with resource availability would lead native trees, dominant in the local vegetation, to be an excellent target for commercialization.

Within the group of commercially important plants, five trees and one shrub were strongly associated with collection in the FLONA, the conservation unit having the role of supplying commercially valuable medicinal plants to the Horizonte residents. Relatively high percentages of collection in anthropogenic areas for some of these trees could indicate some type of species management, as in the case of the 'fava d'anta' tree (Dimorphandra gardneriana Tul.) and 'pequi' (Caryocar coriaceum Wittm.) (Table 3), which are species that provide substantial income for the Horizonte residents. The collection of 'barbatimão' (Stryphnodendron rotundifolium Mart.), 'janaguba' (Himatanthus drasticus (Mart.) Plumel.), 'mangaba' (Hancornia speciosa), and 'catuaba' (Erythroxylum ampliofolium (Mart.) OE Schulz) is prohibited by IBAMA (Instituto Brasileiro do Meio Ambiente e dos Recursos Naturais Renováveis [Brazilian Institute of Environment and Renewable Natural Resources]). Based on the results obtained, one can predict that the collection of these at the commercial level is performed within the FLONA, and thus, these species must be a conservation priority, for which the development of a management plan is recommended.

The commercially valuable parts of cassava (Manihot esculenta Crantz) and 'urucum' (Bixa orellana L.) (Table 3) are also popularly consumed as food, diffusing their medicinal commercial value. Likewise, the commercial value of the two types of oil from 'pequi' is reinforced by their use as food [40]. The 'fava d'anta' tree is considered medicinal by the Horizonte residents primarily because the Merck pharmaceutical industry purchases considerable amounts each year to use in medicines [18]. The uhaloa (Waltheria indica L.), also bought by Merck, was cited by only two informants, indicating that this plant has little commercial importance for the community.

There was no correlation between the commercial values of the plants and their use values ( $\mathrm{rs}=-0.193$, $\mathrm{p}>0.05$ ), a correlation that was also non-significant ( $\mathrm{rs}=-0.032, \mathrm{p}>0.05)$ for the native pharmacopoeia.
This correlation, negative in both cases, indicates that there are plants with high use values and few citations for commercial values (see Table 1), suggesting that the economic importance of medicinal plants, regardless of their versatility, can be related to other factors, i.e., the medicinal effectiveness for which the plant is indicated can result in a high commercial demand, or the low incidence of the disease they treat would result in lower commercial demand.

\section{Conclusions}

From the perspective of its biochemical fundamentals, the apparency hypothesis does not have predictive potential to explain the use value and commercial value of medicinal plants. In other hand, the herbaceous habit showed the highest ethnospecies richness in the community pharmacopeia, which is an expected prediction, corroborating the apparency hypothesis.

Exotic plants have a strong influence on this pharmacopoeia because these plants are determinants of the greater prominence of the herbaceous habit and its practical value as well as the importance of anthropogenic areas. Moreover, the exotic plants are as versatile as native plants in treating diseases.

The FLONA has demonstrated its role as a source of commercially valuable medicinal plants for the Horizonte residents. Moreover, conservation units such as the FLONA are considered to play an important role in conserving the local knowledge of native medicinal plants because excluding exotic plants showed that the native pharmacopoeia is mostly woody and from native vegetation sites.

The results found here, in agreement with those obtained by other authors, indicate that within a pharmacopeia, there are ecological, biochemical, and cultural variables that interact and determine the use and importance of plants in different cognitive and applied aspects. More studies in different ecosystems that include environmental and cultural variables are necessary to increase our understanding of the behavior of tropical pharmacopoeias in relation to the apparency hypothesis.

\section{Competing interests}

The authors declare that they have no competing interests.

\section{Authors' contributions}

AL was the main author responsible for conducting the study, obtaining and analyzing data, and writing the manuscript. Collection of the plant specimens was also carried out by AL, and UPA assisted with identifications. UPA, MFTM and ELA contributed to the design of the study, interpretation of the findings and preparation of the manuscript. All authors read and approved the final manuscript.

\section{Acknowledgements}

We thank the Horizonte residents for their willingness to assist and interest in the project, especially Edilma Cordeiro da Silva for her valuable support in the community. We also thank the following researchers from the Laboratory of Applied and Theoretical Ethnobiology (LEA) for their collaboration in 
multiple activities of the study: Ivanilda Soares, Letícia Zenóbia, Rosemary Sousa, Rafael Domingos, Maria Clara Cavalcanti, Caroline Gomes, Lucilene Lima, Thiago Araújo, Ribamar Junior, Gilney Charll, Alyson Almeida, Rafael Silva, Washington Soares, Patrícia Muniz, Taline Silva, and Mariana Giraldi, and all the others who gave their support in some way. Thanks to Luisa Casas for her help in the field and for reviewing the document. We thank the staff of the herbarium at the Regional University of Cariri (Universidade Regional do Cariri - URCA), Crato Ceará, especially Dr. Maria Arlene Pessoa da Silva, the herbarium director and M.Sc. Ana Cleide Alcântara Morais for her availability and work in identifying the samples. We thank the ICMbio (Instituto Chico Mendes de Conservação da Biodiversidade ['Chico Mendes' Institute for Biodiversity Conservation]) for permission to study the site and CAPES (Coordenação de Aperfeiçoamento de Pessoal de Nível Superior [Coordination for the Improvement of Higher Education Personnel]) for financial support to AL. This paper is contribution P013 of the Rede de Investigação em Biodiversidade e Saberes Locais (REBISA-Network of Research in Biodiversity and Local Knowledge), with financial support from FACEPE (Foundation for Support of Science and Technology) to the project Núcleo de Pesquisa em Ecologia, conservação e Potencial de Uso de Recursos Biológicos no Semiárido do Nordeste do Brasil (Center for Research in Ecology, Conservation and Potential Use of Biological Resources in the Semi-Arid Region of Northeastern Brazil-APQ1264-2.05/10). Also, we thank the anonymous reviewers for their constructive comments, which helped us to improve the manuscript.

\section{Author details}

'Departamento de Biologia, Área de Botânica, Laboratório de Etnobiologia Aplicada e Teórica (LEA), Universidade Federal Rural de Pernambuco, Rua Dom Manoel de Medeiros s/n, Dois Irmãos, Recife 52171-030, Pernambuco, Brasil. 'Departamento de Biologia, Área de Botânica, Laboratório de Ecologia de Ecossistemas Nordestinos, Universidade Federal Rural de Pernambuco, Rua Dom Manoel de Medeiros s/n, Dois Irmãos, Recife 52171-030, Pernambuco, Brasil. ${ }^{3}$ Centro de Educação e Saúde, Universidade Federal de Campina Grande, Campus de Cuité, Olho d'Água da Bica, s/n, Cuité, Paraíba, Brasil.

Received: 2 May 2013 Accepted: 2 January 2014

Published: 10 January 2014

\section{References}

1. Feeny P: Plant Apparency and chemical defense. In Biological Interactions between Plants and Insects. Recent Advances in Phytochemistry. Volume 10. Edited by Wallace JW, Nansel RL. New York: Plenum Press; 1976:1-40.

2. Rhoades DF, Cates RG: Toward a general theory of plant antiherbivore chemistry. In Biological Interactions between Plants and Insects. Recent Advances in Phytochemistry. Volume 10. Edited by Wallace JW, Nansel RL. New York: Plenum Press; 1976:169-213.

3. Albuquerque UP, Lucena RFP: Can apparency afect the use of plants by local people in tropical forest? Interciencia 2005, 30(8):506-511.

4. Lucena RFP, Araújo EL, Albuquerque UP: Does the local availability of woody Caatinga plants (northeastern Brazil) explain their use value? Econ bot 2007, 61(4):347-361.

5. Lucena RFP, Medeiros PM, Araújo EL, Alves AGC, Albuquerque UP: The ecological apparency hypothesis and the importance of useful plants in rural communities from northeastern Brazil: an assessment based on use value. J Enviro Manage 2012, 96:106-115.

6. Albuqueque UP, Ramos MA, Melo JG: New strategies for drug discovery in tropical forests based on ethnobotanical and chemical ecological studies. J Ethnopharmacol 2012, 140:197-201.

7. Chazdon RL, Coe FG: Ethnobotany of woody species in second-growth, old-growth, and selectively logged forests of northeastern Costa Rica. Conserv Biol 1999, 13(6):1312-1322.

8. Gavin MC: Conservation implications of rainforest use patterns: mature forests provide more resources but secondary forests supply more medicine. J Appl Ecol 2009, 46:1275-1282.

9. Thomas E, Van Damme P: Plant use and management in homegardens and swiddens: evidence from the Bolivian Amazon. Agrofor Syst 2010, 80:131-152

10. Bennett $B C$, Prance $G T$ : Introduced plants in the indigenus farmacopedia of northern South America. Econ bot 2000, 54(1):90-102.

11. Stepp JR, Moerman DE: The importance of weeds in ethnopharmacology. J Ethnopharmacol 2001, 75:19-23.
12. Voeks RA: Tropical forest healers and habitat preference. Econ bot 1996, 50(4):381-400.

13. Voeks RA: Disturbance pharmacopoeias: medicine and myth from the humid tropics. Ann Assoc Am Geogr 2004, 94(4):868-888.

14. Phillips O, Gentry AH: The useful plants of Tambopata, Peru: I. Statistical hypothesis test with a new quantitative technique. Econ bot 1993, 47 (1):15-32.

15. Albuquerque UP: Re-examining hypotheses concerning the use and knowledge of medicinal plants: a study in the Caatinga vegetation of NE Brazil. J Ethnobiol Ethnomed 2006, 2:30.

16. La Torre-Cuadros MLA, Islebe GA: Traditional ecological knowledge and use of vegetation in southeastern Mexico: a case study from Solferino, Quintana Roo. Biodive Conserv 2003, 2:2455-2476.

17. Albuquerque UP, Oliveira RF: Is the use-impact on native Caatinga species in Brazil reduced by species richness of medicinal plants? J Ethnopharmacol 2007, 113:156-170.

18. IBAMA: Plano de Manejo da Floresta Nacional do Araripe. Brasília: Instituto Brasileiro do Meio Ambiente e dos Recursos Naturais Renováveis; 2004

19. Albuquerque UP, Lucena RFP, Alencar NL: Methods and techniques used to collect ethnobiological data. In Methods and Techniques in Ethnobiology and Ethnoecology. Edited by Albuquerque UP, Lucena RFP, Cunha L, Alves RRN. New York: Springer; 2014.

20. Begon M, Townsend CR, Harper JL: Ecology: From Individuals to Ecosystems. 4th edition. Oxford: Blackwell Publishing Ltd; 2006.

21. Costa IR, Araújo FS: Organização comunitária de um encrave de Cerrado sensu stricto no bioma Caatinga, chapada do Araripe, Barbalha, Ceará. Acta Bot Bras 2007, 21(2):281-291.

22. Rossato SC, Leitão-Filho HF, Begossi A: Ethnobotany of Caiçaras of the Atlantic Forest coast (Brazil). Econ bot 1999, 53:387-395.

23. Borgatti SP, Natick MA: Anthropac 4.0. Natick: Analytic Technologies; 1996.

24. Ayres M, Ayres JM, Ayres DL, Santos AA: BioEstat: Aplicaçōes Estatísticas nas Areas das Ciências Biológicas e Médicas. Belém: Sociedade Civil Mamirauá: MCT-CNPq; 2007

25. Albuquerque UP, Medeiros PM, Almeida ALS, Monteiro JM, Lins-Neto EMF, Melo JG, Santos JP: Medicinal plants of the Caatinga (semi-arid) vegetation of NE Brazil: a quantitative approach. J Ethnopharmacol 2007, 114:325-354.

26. Almeida CFCBR, Silva TC L e, Amorim ELC, De S, Maia MB, Albuquerque UP: Life strategy and chemical composition as predictors of the selection of medicinal plants from the Caatinga (northeast Brazil). J Arid Environ 2005, 62:127-142.

27. Phillips O, Gentry AH: The useful plants of Tambopata, Peru: II. Additional hypothesis testing in quantitative ethnobotany. Econ bot 1993, 47(1):33-43.

28. Caniago I, Siebert ST: Medicinal plant ecology knowledge and conservation in Kalimantan Indonesia. Econ bot 1998, 52(3):229-250.

29. Gavin MC: Changes in forest use value through ecological succession and their implications for land management in the Peruvian Amazon Conserv Biol 2004, 18(6):1562-1570.

30. Thomas E, Vandebroek I, Van Damme P: Valuation of forests and plant species in indigenous territory and national park Isiboro-Sécure, Bolivia. Econ bot 2009, 63(3):229-241

31. Monteiro JM, Albuquerque UP, Lins-Neto EMF, Araújo EL, Amorim ELC: Use patterns and knowledge of medicinal species among two rural communities in brazil's semi-arid northeastern region. J Ethnopharmacol 2006, 105:173-186.

32. Medeiros MFT, Albuquerque UP: The pharmacy of the Benedictine monks: the use of medicinal plants in northeast Brazil during the nineteenth century (1823-1829). J Ethnopharmacol 2012, 139:280-286.

33. Marques VRB: Natureza em boiōes: medicinas e boticários no Brasil setecentista. Campinas: Editora da Unicamp; 1999.

34. Baider C, Tabarelli M, Mantovani W: O banco de sementes de um trecho de floresta atlântica montana (São Paulo, Brasil). Rev Bras Biol 1999, 59(2):319-328.

35. Ratter JA, Ribeiro JF, Bridgewater S: The brazilian Cerrado vegetation and threats to its biodiversity. Ann Bot 1997, 80:223-230.

36. Frei B, Sticher O, Heinrich M: Zapotec and mixe use of tropical habitats for securing medicinal plants in Mexico. Econ bot 2000, 54(1):73-81.

37. Alencar NL, De Sousa Araújo TA, Cavalcanti De Amorim EL, Albuquerque UP: The inclusion and selection of medicinal plants in traditional pharmacopoeias-evidence in support of the diversification. Econ bot 2010, 64(1):68-79. 
38. Shanley $P$, Luz $L:$ The impacts of forest degradation on medicinal plant use and implications for health care in eastern Amazonia. Bioscience 2003, 53(6):573-584.

39. Monteiro JM, Araújo EL, Amorim ELC, Albuquerque UP: Local markets and the commerce of medicinal plants: a review with emphasis in Brazil. Econ bot 2010, 64(4):352-366.

40. Gonçalves CU: Os piquizeiros da Chapada do Araripe, 25(1), Revista de Geografia. Recife: UFPE - DCG/NAPA; 2008:88-103.

doi:10.1186/1746-4269-10-2

Cite this article as: Lozano et al:: The apparency hypothesis applied to a local pharmacopoeia in the Brazilian northeast. Journal of Ethnobiology and Ethnomedicine 2014 10:2.

\section{Submit your next manuscript to BioMed Central and take full advantage of:}

- Convenient online submission

- Thorough peer review

- No space constraints or color figure charges

- Immediate publication on acceptance

- Inclusion in PubMed, CAS, Scopus and Google Scholar

- Research which is freely available for redistribution 